\title{
Virulence of the Lyme disease spirochete before and after the tick bloodmeal: a quantitative assessment
}

\author{
Irene N. Kasumba ${ }^{1,2}$, Aaron Bestor $^{1}$, Kit Tilly ${ }^{1}$ and Patricia A. Rosa ${ }^{1 *}$
}

\begin{abstract}
Background: Borrelia burgdorferi, the tick-transmitted agent of Lyme disease, adapts to different environments as it cycles between an arthropod vector and vertebrate host. Signals encountered during nymphal tick feeding prior to transmission activate a regulon that is controlled by the alternative sigma factors RpoN and RpoS, which are required for mammalian infection. The ingested bloodmeal also provides nutrients that stimulate spirochete replication. Although the influence of tick feeding on spirochete growth and gene expression is well documented, a quantitative assessment of spirochete virulence before and after tick feeding has not been made.

Methods: Homogenates were prepared from unfed and fed infected Ixodes scapularis nymphs that had acquired $B$. burgdorferi as larvae. Serially diluted tick homogenates were needle-inoculated into mice to determine the infectious dose of tick-derived spirochetes before and after the bloodmeal. Mouse infection was assessed by sero-reactivity with B. burgdorferi whole cell lysates on immunoblots and attempted isolation of spirochetes from mouse tissues. Viable spirochetes in tick-derived inocula were quantified by colony formation in solid media.
\end{abstract}

Results: We found that an inoculum containing as many as $10^{4} \mathrm{~B}$. burgdorferi from unfed ticks is largely non-infectious, while the calculated $I D_{50}$ for spirochetes from fed ticks is $\sim 30$ organisms. Engineered constitutive production of the essential virulence factor OspC by spirochetes within unfed ticks did not confer an infectious phenotype.

Conclusion: Conditional priming of B. burgdorferi during tick feeding induces changes in addition to OspC that are required for infection of the mammalian host.

Keywords: Borrelia burgdorferi, Ixodes ticks, Conditional priming, Lyme disease spirochete

\section{Background}

Borrelia burgdorferi, the spirochetal agent of Lyme borreliosis, is maintained in a natural infectious cycle involving small rodents and Ixodes ticks [1-4]. As $B$. burgdorferi cycles between vector and host, it senses external cues and adapts by making gene products appropriate for each environment (reviewed in $[5,6])$. This ability to detect sudden changes in external stimuli and modulate gene expression is mediated by a relatively small set of known regulatory proteins and sigma factors [7]. Sigma $70\left(\sigma^{70}, \mathrm{RpoD}\right)$ directs the expression of most $B$. burgdorferi genes, including some that are tightly

\footnotetext{
* Correspondence: prosa@niaid.nih.gov

'Laboratory of Zoonotic Pathogens, Rocky Mountain Laboratories, National Institute of Allergy and Infectious Diseases, National Institutes of Health, Hamilton, MT 59840, USA

Full list of author information is available at the end of the article
}

regulated at various stages of the infectious cycle. Two alternative sigma factors, $\sigma^{54}(\mathrm{RpoN})$ and $\sigma^{\mathrm{s}}$ (RpoS), function in concert to direct the expression of a smaller set of spirochete genes required primarily in the mammalian host [8-12].

The RpoN-RpoS regulon is modulated as B. burgdorferi traverses between the mammalian host and tick vector. When larvae feed on an infected mammal and acquire spirochetes, the RpoN-RpoS cascade is deactivated; it is re-activated when molted nymphs take a blood meal and spirochetes in the tick midgut sense accompanying stimuli, such as an influx of nutrients and changes in temperature and $\mathrm{pH}$ (reviewed in [5]). One hallmark of the RpoS-dependent changes that occur during tick feeding is induction of OspC $[8,13]$. OspC is an outer surface lipoprotein that is required by $B$. 
burgdorferi to initiate infection of the mammalian host, but subsequently down regulated during persistent infection [14, 15]. Because of the direct link between activation of the RpoN-RpoS regulon and synthesis of factors required for tick transmission [16] and host infection $[8,14,15,17-19]$, it is anticipated that spirochetes residing in distinct environments within the tick vector (unfed versus fed) will also display significant differences in mammalian infectivity.

An early study by Piesman and colleagues found that unfed nymphal tick homogenates were non-infectious when inoculated into mice and concluded that unfed ticks contained too few spirochetes to constitute an infectious dose [20]. Although these and more recent studies clearly document the influence of stimuli within the starved and fed tick vector on spirochete physiology [20-33], the impact of these factors on spirochete infectivity in the mammalian host has not been quantitatively assessed.

In this study, we directly compared the infectious dose in mice by needle inoculation of defined numbers of viable spirochetes derived from ticks before and after blood feeding. We use the term "attenuated" to refer to spirochetes that are genetically identical to wild-type organisms but exhibit reduced infectivity because of their biological state. We will refer to the event that elicits infectivity in attenuated spirochetes as "conditional priming". Our results demonstrate that viable B. burgdorferi in unfed ticks are highly attenuated in their ability to infect mice, even with an inoculum of $>7 \times 10^{3}$ organisms engineered to constitutively produce the virulence factor OspC. We conclude that conditional priming of B. burgdorferi during tick feeding induces critical changes, in addition to OspC production, that specifically prepare the spirochete for infection of the mammalian host.

\section{Methods}

\section{Borrelia burgdorferi strains and culture conditions} Infectious clone B31-A3, derived from the B31 type strain of B. burgdorferi (ATCC 35210), was used as the wild type (WT) strain, and an isogenic derivative carrying the osp $C$ gene driven by the constitutive $f l a B$ promoter on the shuttle vector $\mathrm{pBSV} 2 \mathrm{G}$, termed $\mathrm{A} 3 /$ fla $B_{\mathrm{p}}:: o s p C$, was used for the constitutive $\operatorname{osp} C$ expression experiments [34, 35]. $B$. burgdorferi liquid cultures were propagated from frozen stocks in Barbour-Stoenner-Kelly II (BSK II) medium containing gelatin and $6 \%$ rabbit serum, and supplemented with $40 \mu \mathrm{g} / \mathrm{ml}$ gentamicin, where appropriate. Viable spirochetes were quantified as colony forming units (CFUs) in solid BSK medium incubated at $35{ }^{\circ} \mathrm{C}$ with $2.5 \% \mathrm{CO}_{2}$, as described previously [36].

\section{Tick infection and infectious dose in mice}

Mouse infection studies utilized 6-to-8 week old female mice of an outbred derivative of Swiss-Webster mice (termed RML) reared at the Rocky Mountain Laboratories breeding facility. Larval ticks acquired spirochetes by feeding on mice infected with WT spirochetes, as described previously [37]. Experiments to determine the $50 \%$ infectious dose $\left(\mathrm{ID}_{50}\right)$ in mice of WT spirochetes derived from unfed and fed infected nymphal ticks were conducted as previously described with minor modifications [37], and calculated by the method of Reed and Muench [38]. Briefly, two groups of 10 nymphs, before or 7 days after feeding on a naïve mouse, were surface sterilized by immersion first in $3 \%$ hydrogen peroxide and then in $70 \%$ ethanol, briefly air dried and subsequently ground in $1 \mathrm{ml}$ BSK medium in a sterile $1.5 \mathrm{ml}$ microfuge tube with a disposable plastic pestle (Kimble Chase, Rockwood, TN). This experiment was performed a second time using 30 unfed nymphs. For each group of fed or unfed nymphs, aliquots of undiluted tick homogenates were inoculated intradermally into mice using $100 \mu \mathrm{l}$ per mouse. The remaining tick homogenates were serially diluted (range of $10^{-1}$ to $10^{-5}$ ), and $100 \mu \mathrm{l}$ of each dilution was inoculated intradermally into mice. Aliquots of tick homogenates were also plated to enumerate $\mathrm{CFU}$ in the inocula. $B$. burgdorferi infection in mice was assessed by seroconversion 3 weeks and isolation of spirochetes from cultures of ear, bladder and joint tissues 5 weeks after inoculation.

A second group of larval ticks was artificially infected by immersion [39] in cultures of WT or A3/fla $B_{\mathrm{p}}:: \operatorname{ospC}$ $B$. burgdorferi; this route of infection was utilized because spirochetes that constitutively produce OspC are either cleared by the adaptive immune response of mice or persist as variants that no longer make OspC [40]. Following the molt, groups of 5-10 nymphs were ground in medium before or immediately after feeding to repletion on naïve mice, and the homogenates were aliquoted and frozen at $-80{ }^{\circ} \mathrm{C}$. At the start of animal experiments, aliquots were thawed and inocula targeting an approximate low $\left(10^{2}\right)$ and high $\left(10^{4}\right)$ spirochete dose per animal were injected into groups of mice. An aliquot of the unfed tick homogenate was also cultivated in medium for four days at $35^{\circ} \mathrm{C}$ prior to inoculation; spirochete density expanded from $\sim 10^{5}$ cells $/ \mathrm{ml}$ to $8 \times 10^{7}$ cells $/ \mathrm{ml}$ during this incubation period. Throughout this study, diluted aliquots of tick homogenates were plated before freezing and at the time of animal inoculation to determine the CFUs in homogenates and inocula. There was no significant loss of viability following freeze/thawing of tick homogenates. Individual unfed and fed ticks from these same cohorts were also homogenized and aliquots plated to determine spirochete burden per tick. B. burgdorferi infection in mice was assessed by sero-conversion 3 weeks and spirochete isolation from tissues 5 weeks after inoculation. 


\section{Ethical approval}

Animal experiments were conducted following guidelines from the National Institutes of Health with protocols approved by the Rocky Mountain Laboratories Animal Care and Use Committee. The Rocky Mountain Laboratories are accredited by the International Association for Assessment and Accreditation of Laboratory Animal Care (AAALAC).

\section{Seroreactivity}

Whole cell lysates of $B$. burgdorferi cultivated to late exponential phase $\left(\sim 7 \times 10^{7}\right.$ spirochetes $\left./ \mathrm{ml}\right)$ were separated by polyacrylamide gel electrophoresis and transferred to nitrocellulose membranes following standard protocols [34]. A slot blot apparatus (Bio-Rad, Hercules, CA) was used to assess reactivity of individual mouse sera with $B$. burgdorferi proteins at 1:200 dilution, as described previously [37].

\section{Immunofluorescence assay (IFA)}

The synthesis of OspC by WT or A3/fla $B_{\mathrm{p}}:: \operatorname{osp} C$ spirochetes in unfed nymphal tick midguts was investigated by IFA and epifluorescence microscopy. Midgut tissues, dissected from unfed nymphs artificially infected as larvae [39] with WT or $\mathrm{A} 3 /$ fla $_{\mathrm{p}}$ ::ospC spirochetes as described above, were fixed and incubated with a mixture of rabbit anti-B. burgdorferi polyclonal serum (provided by Tom Schwan [41]) and anti-OspC monoclonal antibody (provided by Robert Gilmore, [42]). Total Borrelia present and spirochetes synthesizing OspC were detected with a mixture of secondary antibodies containing rhodamine-conjugated goat anti-rabbit and FITC-labeled sheep anti-mouse, respectively.

\section{Statistical analysis}

The statistical significance of differences between spirochete loads in ticks (Fig. 1) was assessed using the GraphPad PRISM software with two-tailed, unpaired
Student's t-test with $95 \%$ confidence interval $(P<0.05)$. The statistical significance of differences between mouse infections with unfed and fed tick homogenates (Tables 1 and 2) was assessed with pooled data using GraphPad software to compute two-tailed $P$ values from Fisher's exact test.

\section{Results}

The $I D_{50}$ in mice varies with $B$. burgdorferi source

Our previous studies calculated an $\mathrm{ID}_{50}$ in mice of $\sim 500$ in vitro-cultivated $B$. burgdorferi, while that of bacteria derived from nymphs fed for $72 \mathrm{~h}$ was $\sim 10$ spirochetes [37]. However, we had not determined the $\mathrm{ID}_{50}$ of spirochetes obtained from nymphs prior to feeding (unfed ticks). Other investigators had demonstrated that homogenates derived from unfed nymphs were not infectious for hamsters or mice, and concluded that spirochete replication during tick feeding was required to attain an infectious dose [20,21, 23]. To determine the approximate number of spirochetes from unfed nymphs required to infect a mouse, larval ticks infected by feeding on WT-infected mice were allowed to molt to nymphs, and nymphal ticks were sampled before and after feeding to repletion on naïve mice. Using a dilution series of homogenates prepared from unfed and fed ticks, we challenged mice by needle inoculation with tick-derived B. burgdorferi and assessed infection. Only $1 / 45$ mice became infected following injection of unfed tick homogenates containing from $\sim 10$ to 10,000 spirochetes, whereas $17 / 20$ mice were infected with a comparable range of inocula from fed ticks $(P<0.0001)$ (Table 1). This outcome does not permit an infectious dose estimate for spirochetes in unfed ticks (although it must be greater than $10^{4}$ ), but yields an $\mathrm{ID}_{50}$ of $\sim 30$ organisms for B. burgdorferi derived from replete nymphs 7 days after drop off, similar to previous determinations [37]. These data establish that viable spirochetes in
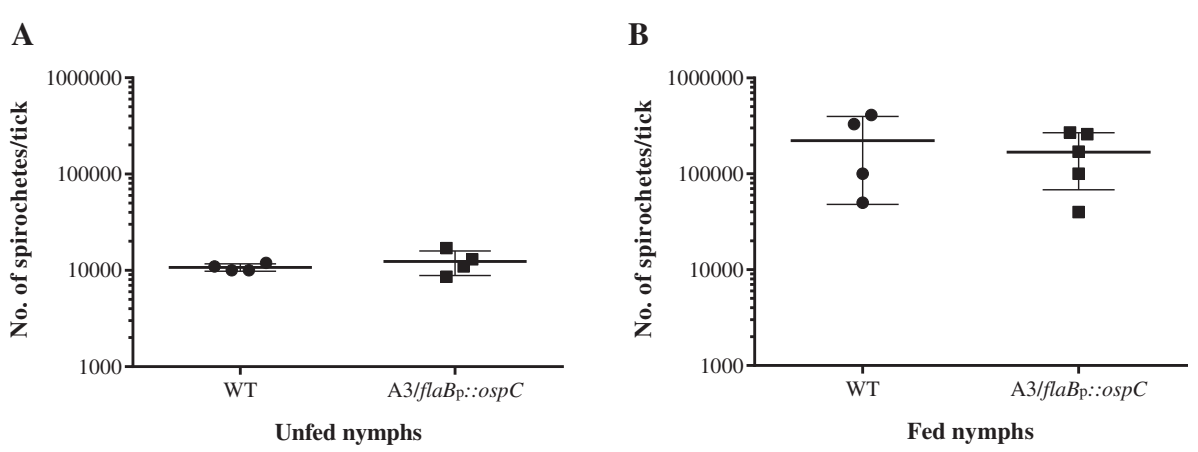

Fig. 1 Spirochete loads in unfed and fed nymphs infected with WT and A3/flaBp::ospC. Nymphs artificially infected as larvae with WT B. burgdorferi or spirochetes engineered to constitutively produce OspC (A3/fla $B_{p}::$ ospC), were homogenized and plated $\mathbf{a}$ before and $\mathbf{b}$ after feeding on naïve mice to determine spirochete loads in ticks. The mean number of spirochetes ( \pm SD) was similar between groups $(P>0.05$, Student's t-test, unpaired, 2-tailed) and increased approximately 10-fold when nymphs fed 
Table 1 Infectious dose in mice of WTB. burgdorferi derived from unfed and fed ticks

\begin{tabular}{|c|c|c|c|}
\hline \multirow[t]{2}{*}{ Spirochete source } & \multirow[b]{2}{*}{$\begin{array}{l}\text { Inoculum }^{\mathrm{a}} \\
\text { (spirochetes/ } \\
\text { mouse) }\end{array}$} & \multicolumn{2}{|l|}{ Mouse infection } \\
\hline & & $\begin{array}{l}\text { Sero-positive mice } \\
\text { (infected/no. } \\
\text { inoculated) }\end{array}$ & $\begin{array}{l}\text { Tissue isolationc } \\
\text { (infected/no. } \\
\text { inoculated) }\end{array}$ \\
\hline \multicolumn{4}{|l|}{ UNFED Ticks $^{d}$} \\
\hline \multirow[t]{3}{*}{ Nymphs (group 1) } & $\sim 1$ & $0 / 5$ & $0 / 5$ \\
\hline & 8 & $0 / 5$ & $0 / 5$ \\
\hline & 80 & $0 / 5$ & $0 / 5$ \\
\hline \multirow[t]{4}{*}{ Nymphs (group 2) } & $\sim 10$ & $0 / 5$ & $0 / 5$ \\
\hline & 110 & $0 / 5$ & $0 / 5$ \\
\hline & 1,100 & $0 / 5$ & $0 / 5$ \\
\hline & 11,000 & $1 / 5$ & $1 / 5$ \\
\hline \multirow[t]{4}{*}{ Adults } & $\sim 1$ & $0 / 5$ & $0 / 5$ \\
\hline & 13 & $0 / 5$ & $0 / 5$ \\
\hline & 130 & $0 / 5$ & $0 / 5$ \\
\hline & 1,300 & $0 / 5$ & $0 / 5$ \\
\hline \multicolumn{4}{|l|}{ FED Ticks ${ }^{e}$} \\
\hline \multirow[t]{5}{*}{ Nymphs } & $\sim 9$ & $0 / 2$ & $0 / 2$ \\
\hline & 90 & $5 / 5$ & $5 / 5$ \\
\hline & 900 & $5 / 5$ & $5 / 5$ \\
\hline & 9,000 & $5 / 5$ & $5 / 5$ \\
\hline & 90,000 & $5 / 5$ & $5 / 5$ \\
\hline
\end{tabular}

${ }^{a}$ No. of $B$. burgdorferi estimated by plating an aliquot of inocula for CFU ${ }^{\mathrm{b}}$ Mice were bled 3 weeks after inoculation and assessed for seroconversion to B. burgdorferi whole cell lysates by immunoblot analysis

${ }^{c}$ Mice were euthanized $5-6$ weeks after inoculation and infection determined by isolation of spirochetes from the ear, joint, and bladder tissues

${ }^{\mathrm{d}}$ Groups of unfed nymphs were homogenized in medium and serially diluted for inoculation into mice (nymph groups (1) and (2) represent pools of 10 and 30 ticks, respectively)

${ }^{\mathrm{e}} \mathrm{A}$ group of 10 nymphs fed to repletion was homogenized in medium 7 days after drop-off and serially diluted for inoculation into mice

unfed ticks are highly attenuated relative to spirochetes obtained from recently fed ticks.

\section{Constitutive production of OspC by spirochetes in unfed ticks does not restore virulence}

The production of $\mathrm{OspC}$, an essential virulence factor for mammalian infection [14], ceases during tick acquisition of B. burgdorferi from an infected mammal and the protein remains undetectable on spirochetes until the subsequent tick blood meal [13, 41]. Because spirochetes derived from unfed ticks were essentially avirulent in mice (Table 1), we wished to determine whether engineered constitutive production of OspC could restore infectivity. To do this, larval ticks were infected by immersion [39] in cultures of WT spirochetes or an isogenic derivative engineered to constitutively produce OspC (A3/fla $\left.B_{\mathrm{p}}:: o s p C\right)$ [35], and then fed to repletion on naïve mice. Larval ticks were allowed to molt and spirochete loads were determined for both cohorts before
Table 2 Infectivity of tick-derived spirochetes engineered to constitutively produce OspC

\begin{tabular}{|c|c|c|c|}
\hline \multirow{2}{*}{$\begin{array}{l}\text { Spirochete source } \\
\text { and strain }\end{array}$} & \multirow[b]{2}{*}{$\begin{array}{l}\text { Inoculum }^{\text {a }} \\
\text { (spirochetes/ } \\
\text { mouse) }\end{array}$} & \multicolumn{2}{|l|}{ Mouse infection } \\
\hline & & $\begin{array}{l}\text { Sero-conversion } \\
\text { (infected/no. } \\
\text { inoculated) }\end{array}$ & $\begin{array}{l}\text { Tissue isolation } \\
\text { (infected/no. } \\
\text { inoculated) }\end{array}$ \\
\hline \multicolumn{4}{|l|}{ UNFED Ticks } \\
\hline \multirow[t]{2}{*}{ WT } & $\sim 90$ & $0 / 5$ & $0 / 5$ \\
\hline & 8,900 & $0 / 5$ & $0 / 5$ \\
\hline \multirow[t]{2}{*}{$A 3 / f l a B_{p}:: O s p C^{d}$} & $\sim 70$ & $0 / 5$ & $0 / 5$ \\
\hline & 7,300 & $0 / 5$ & $0 / 5$ \\
\hline \multicolumn{4}{|l|}{ After Cultivation ${ }^{\text {e }}$} \\
\hline \multirow[t]{2}{*}{ WT } & $\sim 10$ & $0 / 5$ & $0 / 5$ \\
\hline & 1,000 & $5 / 5$ & $5 / 5$ \\
\hline \multicolumn{4}{|l|}{ FED Ticks } \\
\hline \multirow[t]{2}{*}{ WT } & $\sim 190$ & $5 / 5$ & $5 / 5$ \\
\hline & 18,800 & $5 / 5$ & $5 / 5$ \\
\hline \multirow[t]{2}{*}{ A3/fla $B_{p}:: 0 s p C$} & $\sim 90$ & $5 / 5$ & $5 / 5$ \\
\hline & 8,800 & $5 / 5$ & $5 / 5$ \\
\hline
\end{tabular}

${ }^{\mathrm{a}}$ Groups of 5-10 infected ticks were ground in medium before or immediately after feeding to repletion on naïve mice, and homogenates frozen at $-80^{\circ} \mathrm{C}$. Homogenates were thawed for inoculation of mice, and aliquots plated to determine the number of viable spirochetes in each inoculum

${ }^{b}$ Mice were bled 3 weeks post-challenge and sero-conversion to $B$. burgdorferi whole cell lysates assessed by immunoblot analysis

${ }^{c}$ Mice were euthanized $5-6$ weeks post-challenge and infection assessed by attempted isolation of spirochetes from the ear, joint and bladder tissues of each mouse

${ }^{\mathrm{d}} \mathrm{A} 3 / \mathrm{fla}_{\mathrm{p}}$ ::OSpC refers to WT spirochetes engineered to constitutively produce OspC [15]

${ }^{\text {e}}$ Spirochetes in unfed tick homogenates previously frozen at $-80^{\circ} \mathrm{C}$ were thawed and cultivated in BSK II medium for 4 days at $35^{\circ} \mathrm{C}$ prior to mouse inoculation. The number of viable bacteria injected was determined by plating an aliquot of each inoculum

and after the nymphal bloodmeal. There was a 10-fold increase in spirochete loads in nymphs after feeding, and similar numbers of bacteria were detected for both strains $(P>0.05$; Fig. 1$)$, indicating that there was no difference between strains in their ability to colonize, persist and replicate in nymphs. We also assessed OspC production by spirochetes in unfed nymphal tick midguts using immunofluorescence. As expected, none of the WT spirochetes were OspC-positive, whereas all A3/ fla $B_{\mathrm{p}}:: o s p C$ spirochetes present in an unfed nymph made OspC (Fig. 2).

Next, we assessed the infectivity in mice of both groups of tick-derived spirochetes. Using homogenates from unfed or fed infected nymphs, naïve mice were inoculated with approximately $10^{2}$ and $10^{4}$ spirochetes. None of the mice $(0 / 20)$ inoculated with WT or $\mathrm{A} 3 /$ fla $B_{\mathrm{p}}::$ osp $C$ from unfed tick homogenates became infected, whereas all mice (20/20) challenged with fed tick homogenates carrying either strain became infected and spirochetes were isolated from all tissues tested $(P<0.0001)$ (Table 2$)$. We did not 


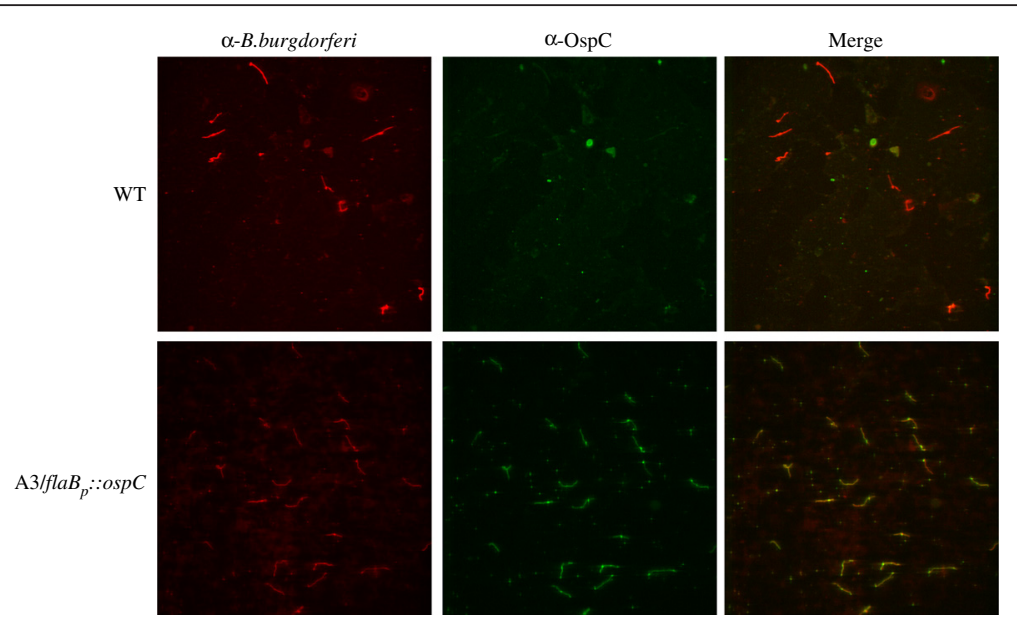

Fig. 2 OspC production by WT and A3/fla $B_{p}:: 0 s p C$ spirochetes in unfed nymphs. Nymphs were artificially infected as larvae with wild-type B. burgdorferi strain B31-A3 (WT) or an isogenic derivative engineered to constitutively express ospC (A3/flaB $B_{\mathrm{p}}:$ :ospC). Spirochetes in dissected tick midguts were detected by IFA with a polyclonal anti-B. burgdorferi primary antiserum [41] and rhodamine-labeled secondary antibody, while synthesis of OspC was examined using a monoclonal anti-OspC primary antibody [42] and FITC-labeled secondary antibody

assess shuttle vector retention in isolates from mice inoculated with $\mathrm{A} 3 / f l a B_{\mathrm{p}}:: o s p C$, but we would expect the constitutively expressed $\operatorname{ssp} C$ gene to be lost in the majority of spirochetes by 5 weeks of infection, as previously demonstrated to be necessary for persistence of these spirochetes after immune recognition of OspC [35, 40, 43]. Additionally, WT organisms that grew out from liquid cultivation of unfed tick homogenates were infectious in mice $(5 / 5)$ with an inoculum of $\sim 10^{3}$ spirochetes (Table 2 ), consistent with previous estimates of infectivity with in vitro grown organisms [37]. Importantly, these data demonstrate that tick feeding primes $B$. burgdorferi for infection in mice by induction of critical components in addition to the known virulence factor OspC.

\section{Discussion}

The Lyme disease spirochete must undergo specific physiological changes in order to adjust and survive in the different environments encountered during its natural infectious cycle (reviewed in [5, 6]). Spirochetes must also migrate from the tick midgut to the salivary glands in order to be transmitted, a process that typically takes $\sim 48 \mathrm{~h}$ $[23,26,44-47]$. We designed the present study to quantitatively assess the virulence of spirochetes derived from ticks before and after feeding on a vertebrate host. We needle-inoculated mice with infected tick material to bypass requirements specific for tick transmission and focus the comparison on the relative capabilities of these spirochetes to infect a mammalian host. Viable spirochetes in these inocula were quantified by colony formation in solid medium. A significant finding of this study was that $0 / 10$ mice were infected with an inoculum of $\sim 10^{3}$ viable spirochetes from unfed ticks and only $1 / 15$ mice became infected with inocula ranging from $\sim 7 \times 10^{3}$ to $1 \times 10^{4}$ organisms from this source (Table 1). This outcome does not permit calculation of the $\mathrm{ID}_{50}$ for spirochetes from unfed ticks, whereas we calculated an $\mathrm{ID}_{50}$ of $\sim 30$ for spirochetes from fully engorged ticks. Increasing the inoculum 10-fold to $10^{5}$ organisms in order to potentially determine an $\mathrm{ID}_{50}$ for spirochetes in unfed ticks would require injecting the undiluted homogenate of $\sim 30$ unfed ticks per mouse (Table 1), which is technically limiting. When the outcomes of separate experiments are considered together (Tables 1 and 2), only 1/65 mice became infected following inoculation of $\sim 10$ to $10^{4}$ spirochetes from unfed ticks, whereas 35/37 mice were infected by similar numbers of tick-derived spirochetes after the bloodmeal. Together these results demonstrate a large increase in the virulence of spirochetes when they have been "primed" by a blood meal. Previous studies have reported the lack of infectivity of B. burgdorferi from unfed ticks, but this outcome was attributed to potentially low numbers of bacteria in the inocula, which were difficult to accurately measure [20, 23]. However, our findings indicate that in addition to physical location and absolute number, the virulence of resident spirochetes is fundamentally different after tick feeding commences.

One well-characterized and major difference between B. burgdorferi in unfed versus fed ticks is the differential production of outer surface proteins, with OspA produced by spirochetes in unfed ticks and OspC induced during tick feeding $[13,41,48]$. The presence of OspA on spirochetes has been correlated with successful colonization of the tick midgut [49-51], while induction of OspC during tick feeding is an absolute requirement for B. burgdorferi 
to initiate infection of the vertebrate host $[14,15]$. Therefore, since $B$. burgdorferi in unfed ticks do not produce $\mathrm{OspC}$, it is perhaps not surprising that spirochetes persisting within an unfed tick environment would be unable to infect mice. However, we demonstrated that spirochetes derived from unfed ticks failed to infect mice even when they were engineered to constitutively produce OspC (Table 2). These data indicate that the presence of the virulence factor OspC is not sufficient to 'prime' spirochetes coming out of unfed ticks for productive mouse infection, and further demonstrate that induction of $\mathrm{OspC}$ is only one of several critical adaptive responses that spirochetes undergo during tick feeding to prepare for host infection [31, 32, 52].

There are plausible explanations for the observed lack or significant attenuation of virulence of spirochetes in the unfed tick vector other than OspC. The RpoN-RpoS regulatory cascade, which governs expression of many $B$. burgdorferi genes in the mammalian host, is shut off during spirochete acquisition by feeding ticks and remains off until it is activated during the next blood meal $[11,12,32,52]$. A recent study by Iyer and colleagues utilized an amplification-microarray approach to compare the transcriptomes of mammalian host-adapted spirochetes with those in fed ticks or cultivated in vitro [31]. Significant differences were noted in the global patterns of gene expression among spirochetes from these distinct sources, particularly in various aspects of metabolism, nutrient uptake and chemotactic response [31]. These spirochetes were all in metabolically active states supported by nutrients present in the host, the fed tick or culture medium, whereas metabolically inactive spirochetes in unfed nymphal ticks (which we found to be non-infectious) were not part of this comparison because they do not provide enough material for microarray analysis. A direct comparison of global gene expression between spirochetes from fed and unfed ticks would be extremely insightful, however, and the substantially larger unfed adult tick, whose spirochete burden is similarly non-infectious (Table 1), but approximately 50 -fold higher than that of an unfed nymph (data not shown), could represent a good source of material for such future analyses.

Genes with RpoS-dependent expression patterns like ospC (abundantly transcribed by spirochetes in the host and in fed nymphal ticks, but expressed at very low levels by spirochetes in fed larval ticks), should provide insight into virulence factors specifically induced for host infection rather than stimulated for cell growth by the bloodmeal [31, 32]. Surprisingly, of the 100 genes most abundantly expressed by spirochetes in fed nymphs, only $o s p C$ exhibited this anticipated pattern of putative virulence factor expression [31]. In addition, only a few members of the previously identified set of RpoS-dependent genes of B. burgdorferi [11] appear in the "top 100" list in fed nymphs, and of these, $o s p C$ is the only gene that is also highly expressed by host-adapted spirochetes [31]. It seems unlikely that metabolic state and OspC production, while both critically important, are the sole determinants of the infectious phenotype of spirochetes in fed versus unfed nymphs. Rather, less abundant gene products not highlighted by microarray analyses are also likely to play a key role in preparing $B$. burgdorferi for mammalian infection. Likewise, transcriptome comparisons do not identify post-transcriptional regulatory mechanisms that alter translation or protein turnover, which have been shown to play a role in modulation of RpoS function [53, 54]. Some spirochetal components, such as the integral outer membrane protein $\mathrm{P} 66$, are induced during tick feeding independently of the RpoN-RpoS regulon, yet are essential in the host $[55,56]$. Finally, other B. burgdorferi factors that are specifically made during the tick starvation period could actively impede spirochete infectivity in the mammalian host $[16,24,25,27,32,53,57,58]$. Thus, both the presence and absence of particular factors could contribute to the avirulent phenotype of spirochetes in unfed ticks.

\section{Conclusions}

Our current study illustrates the impact of the vector environment on the physiological or biological state of the Lyme disease spirochete, which in turn directly influences its virulence in the mammalian host. Spirochete replication as a consequence of the blood meal is clearly important during the natural route of transmission by tick bite. However, our findings demonstrate that a fundamental difference between spirochetes in unfed and fed ticks is their exposure and subsequent response to a key priming event that enables mammalian infection. This study and others (for example [13, 27, 31, 32, 48]) underscore the need for direct analysis of $B$. burgdorferi within the tick vector throughout the transmission cycle. Such investigations will provide a better understanding of the basic biology of the Lyme disease spirochete, which forms a basis for rational design of preventive and therapeutic interventions for human infection.

\section{Abbreviations \\ WT: wild type; CFU: colony forming unit; Osp: outer surface protein; IFA: immunofluorescence assay; $\mathrm{ID}_{50}$ : dose needed to infect $50 \%$ of challenged recipients.}

\section{Competing interests}

The authors declare that they have no competing interests.

\section{Authors' contributions}

$\mathbb{K}$ and $A B$ prepared the infected ticks, inoculated mice and assessed the outcome. KT assisted with some of the animal infection studies. AB and PR conceived of the study. IK, AB and PR participated in the design of the study and analysis of data. IK and PR drafted the manuscript. All authors read and approved the final manuscript. 


\section{Acknowledgments}

We thank Martha Thayer, David Bland, Crystal Richards, Frank Gherardini, Tom Schwan and Phil Stewart for critical review of the manuscript. We are grateful to Vinod Nair and Anita Mora for assistance with graphics. This research was supported by the Intramural Research Program of the National Institute of Allergy and Infectious Diseases, National Institutes of Health.

\section{Author details}

'Laboratory of Zoonotic Pathogens, Rocky Mountain Laboratories, National Institute of Allergy and Infectious Diseases, National Institutes of Health, Hamilton, MT 59840, USA. ${ }^{2}$ Current address: Center for Vaccine Development, Department of Geographic Medicine, University of Maryland School of Medicine, Baltimore, MD 21201, USA.

\section{Received: 14 December 2015 Accepted: 12 February 2016 Published online: 07 March 2016}

\section{References}

1. Burgdorfer W, Barbour AG, Hayes SF, Benach JL, Grunwaldt E, Davis JP. Lyme disease - a tick-borne spirochetosis? Science. 1982;216:1317-9.

2. Burgdorfer W, Hayes SF, Benach JL. Development of Borrelia burgdorferi in ixodid tick vectors. In: Benach $\mathrm{J}$, Bosler EM, editors. Lyme disease and related disorders. New York: New York Academy of Sciences; 1988. p. 172-9.

3. Donahue JG, Piesman J, Spielman A. Reservoir competence of white-footed mice for Lyme disease spirochetes. Am J Trop Med Hyg. 1987;36:92-6.

4. Lindsay LR, Barker IK, Surgeoner GA, McEwen SA, Campbell GD. Duration of Borrelia burgdorferi infectivity in white-footed mice for the tick vector Ixodes scapularis under laboratory and field conditions in Ontario. J Wildl Dis. 1997;33(4):766-75

5. Radolf JD, Caimano MJ, Stevenson B, Hu LT. Of ticks, mice and men: understanding the dual-host lifestyle of Lyme disease spirochaetes. Nat Rev Microbiol. 2012;10(2):87-99.

6. Samuels DS. Gene regulation in Borrelia burgdorferi. Annu Rev Microbiol. 2011;65:479-99.

7. Fraser CM, Casjens S, Huang WM, Sutton GG, Clayton $\mathrm{R}$, Lathigra $\mathrm{R}$, White $\mathrm{O}$, Ketchum KA, Dodson R, Hickey EK, et al. Genomic sequence of a Lyme disease spirochaete, Borrelia burgdorferi. Nature. 1997:390:580-6.

8. Hübner A, Wang X, Nolen DM, Popova TG, Cabello FC, Norgard M. Expression of Borrelia burgdorferi OspC and DbpA is controlled by a RpoN-RpoS regulatory pathway. Proc Natl Acad Sci U S A. 2001;98(22):12724-9.

9. Fisher MA, Grimm D, Henion AK, Elias AF, Stewart PE, Rosa PA, Gherardini FC. Borrelia burgdorferi sigma54 is required for mammalian infection and vector transmission but not for tick colonization. Proc Natl Acad Sci U S A. 2005;102(14):5162-7.

10. Caimano MJ, Eggers CH, Gonzalez CA, Radolf JD. Alternate sigma factor RpoS is required for the in vivo-specific repression of Borrelia burgdorferi plasmid Ip54-borne ospA and Ip6.6 genes. J Bacteriol. 2005;187(22):7845-52.

11. Caimano MJ, Iyer R, Eggers CH, Gonzalez C, Morton EA, Gilbert MA, Schwartz I, Radolf JD. Analysis of the RpoS regulon in Borrelia burgdorferi in response to mammalian host signals provides insight into RpoS function during the enzootic cycle. Mol Microbiol. 2007;65(5):1193-217.

12. Ouyang Z, Narasimhan S, Neelakanta G, Kumar M, Pal U, Fikrig E, Norgard MV. Activation of the RpoN-RpoS regulatory pathway during the enzootic life cycle of Borrelia burgdorferi. BMC Microbiol. 2012;12:44.

13. Schwan TG, Piesman J, Golde WT, Dolan MC, Rosa PA. Induction of an outer surface protein on Borrelia burgdorferi during tick feeding. Proc Natl Acad Sci U S A. 1995;92:2909-13.

14. Grimm D, Tilly K, Byram R, Stewart PE, Krum JG, Bueschel DM, Schwan TG, Policastro PF, Elias AF, Rosa PA. Outer-surface protein C of the Lyme disease spirochete: A protein induced in ticks for infection of mammals. Proc Natl Acad Sci U S A. 2004;101(9):3142-7.

15. Tilly K, Krum JG, Bestor A, Jewett MW, Grimm D, Bueschel D, Byram R, Dorward D, Stewart P, Rosa P. Borrelia burgdorferi OspC protein required exclusively in a crucial early stage of mammalian infection. Infect Immun. 2006;74(6):3554-64.

16. Dunham-Ems SM, Caimano MJ, Eggers $\mathrm{CH}$, Radolf JD. Borrelia burgdorferi requires the alternative sigma factor RpoS for dissemination within the vector during tick-to-mammal transmission. PLoS Pathog. 2012;8(2):e1002532

17. Caimano MJ, Eggers $\mathrm{CH}$, Hazlett KR, Radolf JD. RpoS is not central to the general stress response in Borrelia burgdorferi but does control expression of one or more essential virulence determinants. Infect Immun. 2004;72:6433-45.
18. Pal U, Yang X, Chen M, Bockenstedt LK, Anderson JF, Flavell RA, Norgard MV, Fikrig E. OspC facilitates Borrelia burgdorferi invasion of Ixodes scapularis salivary glands. J Clin Invest. 2004;113(2):220-30.

19. Ouyang Z, Kumar M, Kariu T, Haq S, Goldberg M, Pal U, Norgard MV. BosR (BB0647) governs virulence expression in Borrelia burgdorferi. Mol Microbiol. 2009;74(6):1331-43.

20. Piesman J. Dynamics of Borrelia burgdorferi transmission by nymphal Ixodes dammini ticks. J Infect Dis. 1993;167:1082-5.

21. Piesman J, Oliver JR, Sinsky RJ. Growth kinetics of the Lyme disease spirochete (Borrelia burgdorferi) in vector ticks (Ixodes dammini). Am J Trop Med Hyg. 1990;42:352-7.

22. de Silva AM, Fikrig E. Growth and migration of Borrelia burgdorferi in Ixodes ticks during blood feeding. Am J Trop Med Hyg. 1995;53:397-404

23. Crippa M, Rais O, Gern L. Investigations on the mode and dynamics of transmission and infectivity of Borrelia burgdorferi sensu stricto and Borrelia afzelii in Ixodes ricinus ticks. Vector Borne Zoonotic Dis. 2002;2(1):3-9.

24. Rogers EA, Terekhova D, Zhang HM, Hovis KM, Schwartz I, Marconi RT. Rrp1, a cyclic-di-GMP-producing response regulator, is an important regulator of Borrelia burgdorferi core cellular functions. Mol Microbiol. 2009;71(6):1551-73.

25. Sultan SZ, Pitzer JE, Miller MR, Motaleb MA. Analysis of a Borrelia burgdorferi phosphodiesterase demonstrates a role for cyclic-di-guanosine monophosphate in motility and virulence. Mol Microbiol. 2010;77(1):128-42.

26. Dunham-Ems SM, Caimano MJ, Pal U, Wolgemuth CW, Eggers $\mathrm{CH}$, Balic A, Radolf JD. Live imaging reveals a biphasic mode of dissemination of Borrelia burgdorferi within ticks. J Clin Invest. 2009;119(12):3652-65.

27. He M, Ouyang Z, Troxell B, Xu H, Piesman J, Norgard MV, Gomelsky M, Yang XF. Cyclic di-GMP is essential for the survival of the Lyme disease spirochete in ticks. PLoS Pathog. 2011;7(6):e1002133.

28. Pappas CJ, lyer R, Petzke MM, Caimano MJ, Radolf JD, Schwartz I. Borrelia burgdorferi requires glycerol for maximum fitness during the tick phase of the enzootic cycle. PLoS Pathog. 2011;7(7):e1002102.

29. Caimano MJ, Kenedy MR, Kairu T, Desrosiers DC, Harman M, Dunham-Ems S, Akins DR, Pal U, Radolf JD. The hybrid histidine kinase Hk1 is part of a twocomponent system that is essential for survival of Borrelia burgdorferi in feeding Ixodes scapularis ticks. Infect Immun. 2011;79(8):3117-30.

30. Kariu T, Smith A, Yang X, Pal U. A chitin deacetylase-like protein is a predominant constituent of tick peritrophic membrane that influences the persistence of Lyme disease pathogens within the vector. PLoS One. 2013:8(10):e78376.

31. Iyer R, Caimano MJ, Luthra A, Axline Jr D, Corona A, lacobas DA, Radolf JD, Schwartz I. Stage-specific global alterations in the transcriptomes of Lyme disease spirochetes during tick feeding and following mammalian host adaptation. Mol Microbiol. 2015;95(3):509-38.

32. Caimano MJ, Dunham-Ems S, Allard AM, Cassera MB, Kenedy M, Radolf JD. Cyclic di-GMP modulates gene expression in Lyme disease spirochetes at the tick-mammal interface to promote spirochete survival during the blood meal and tick-to-mammal transmission. Infect Immun. 2015:83(8):3043-60

33. Drecktrah D, Lybecker M, Popitsch N, Rescheneder P, Hall LS, Samuels DS. The Borrelia burgdorferi ReIA/SpoT homolog and stringent response regulate survival in the tick vector and global gene expression during starvation. PLoS Pathog. 2015;11(9):e1005160.

34. Elias AF, Stewart PE, Grimm D, Caimano MJ, Eggers CH, Tilly K, Bono JL, Akins DR, Radolf JD, Schwan TG. Clonal polymorphism of Borrelia burgdorferi strain B31 Ml: implications for mutagenesis in an infectious strain background. Infect Immun. 2002;70(4):2139-50.

35. Tilly K, Bestor A, Dulebohn DP, Rosa PA. OspC-independent infection and dissemination by host-adapted Borrelia burgdorferi. Infect Immun. 2009;77(7):2672-82.

36. Elias AF, Bono JL, Kupko JJ, Stewart PE, Krum JG, Rosa PA. New antibiotic resistance cassettes suitable for genetic studies in Borrelia burgdorferi. J Mol Microbiol Biotechnol. 2003;6(1):29-40.

37. Bestor A, Rego RO, Tilly K, Rosa PA. Competitive advantage of Borrelia burgdorferi with outer surface protein BBA03 during tick-mediated infection of the mammalian host. Infect Immun. 2012;80(10):3501-11.

38. Reed $\sqcup$, Muench $H$. A simple method of estimating fifty percent endpoints. Am J Hyg. 1938:27(3):493-7.

39. Policastro PF, Schwan TG. Experimental infection of Ixodes scapularis larvae (Acari: Ixodidae) by immersion in low passage cultures of Borrelia burgdorferi. J Med Entomol. 2003;40(3):364-70. 
40. Xu Q, Seemanapalli SV, McShan K, Liang FT. Constitutive expression of outer surface protein C diminishes the ability of Borrelia burgdorferi to evade specific humoral immunity. Infect Immun. 2006;74(9):5177-84.

41. Schwan TG, Piesman J. Temporal changes in outer surface proteins $A$ and $C$ of the Lyme disease-associated spirochete, Borrelia burgdorferi, during the chain of infection in ticks and mice. J Clin Microbiol. 2000;39(1):382-8.

42. Mbow ML, Gilmore Jr RD, Titus RG. An OspC-specific monoclonal antibody passively protects mice from tick-transmitted infection by Borrelia burgdorferi B31. Infect Immun. 1999;67(10):5470-2.

43. Liang FT, Yan J, Mbow ML, Sviat SL, Gilmore RD, Mamula M, Fikrig E. Borrelia burgdorferi changes its surface antigenic expression in response to host immune responses. Infect Immun. 2004;72(10):5759-67.

44. Ribeiro JMC, Mather TN, Piesman J, Spielman A. Dissemination and salivary delivery of Lyme disease spirochetes in vector ticks (Acari: Ixodidae). J Med Entomol. 1987:24:201-5.

45. Piesman J, Mather TM, Sinsky RJ, Spielman A. Duration of tick attachment and Borrelia burgdorferi transmission. J Clin Microbiol. 1987;25:557-8.

46. Zung JL, Lewengrub S, Rudzinska MA, Spielman A, Telford SR, Piesman J. Fine structural evidence for the penetration of the Lyme disease spirochete Borrelia burgdorferi through the gut and salivary tissues of Ixodes dammini. Can J Zool. 1989;67:1737-48.

47. Piesman J. Dispersal of the Lyme disease spirochete Borrelia burgdorferi to salivary glands of feeding nymphal Ixodes scapularis (Acari: Ixodidae). J Med Entomol. 1995;32:519-21.

48. Ohnishi J, Piesman J, de Silva AM. Antigenic and genetic heterogeneity of Borrelia burgdorferi populations transmitted by ticks. Proc Natl Acad Sci U S A. 2001;98(2):670-5

49. Yang XF, Pal U, Alani SM, Fikrig E, Norgard MV. Essential role for OspA/B in the life cycle of the Lyme disease spirochete. J Exp Med. 2004;199(5):641-8.

50. Pal U, Li X, Wang T, Montgomery RR, Ramamoorthi N, de Silva AM, Bao F, Yang X, Pypaert M, Pradhan D, et al. TROSPA, an Ixodes scapularis receptor for Borrelia burgdorferi. Cell. 2004;119(4):457-68.

51. Battisti JM, Bono JL, Rosa PA, Schrumpf ME, Schwan TG, Policastro PF. Outer surface protein A protects Lyme disease spirochetes from acquired host immunity in the tick vector. Infect Immun. 2008;76(11):5228-37.

52. Xu Q, Shi Y, Dadhwal P, Liang FT. Rpos regulates essential virulence factors remaining to be identified in Borrelia burgdorferi. PLoS One. 2012:7(12):e53212

53. Dulebohn DP, Hayes BM, Rosa PA. Global repression of host-associated genes of the Lyme disease spirochete through post-transcriptional modulation of the alternative sigma factor RpoS. PLoS One. 2014;9(3):e93141.

54. Lybecker MC, Samuels DS. Temperature-induced regulation of RpoS by a small RNA in Borrelia burgdorferi. Mol Microbiol. 2007;64:1075-89.

55. Cugini C, Medrano M, Schwan TG, Coburn J. Regulation of expression of the Borrelia burgdorferi beta(3)-chain integrin ligand, P66, in ticks and in culture. Infect Immun. 2003;71(2):1001-7.

56. Ristow LC, Miller HE, Padmore LJ, Chettri R, Salzman N, Caimano MJ, Rosa PA Coburn J. The beta(3)-integrin ligand of Borrelia burgdorferi is critical for infection of mice but not ticks. Mol Microbiol. 2012:85(6):1105-18.

57. Sze CW, Smith A, Choi YH, Yang X, Pal U, Yu A, Li C. Study of the response regulator Rrp1 reveals its regulatory role in chitobiose utilization and virulence of Borrelia burgdorferi. Infect Immun. 2013;81(5):1775-87.

58. Hayes BM, Dulebohn DP, Sarkar A, Tilly K, Bestor A, Ambroggio X, Rosa PA Regulatory protein BBD18 of the lyme disease spirochete: essential role during tick acquisition? MBio. 2014;5(2):e01017-01014.

\section{Submit your next manuscript to BioMed Central and we will help you at every step:}

- We accept pre-submission inquiries

- Our selector tool helps you to find the most relevant journal

- We provide round the clock customer support

- Convenient online submission

- Thorough peer review

- Inclusion in PubMed and all major indexing services

- Maximum visibility for your research

Submit your manuscript at www.biomedcentral.com/submit

) Biomed Central 